Relations industrielles

Industrial Relations

\title{
Collective Bargaining in Government (Readings and Cases) by J. Joseph Loewenberg and Michel H. Moskow, Englewood Cliffs, New Jersey, Prentice-Hall, Inc., 1972, 362 pp.
}

\section{Jean Boivin}

Volume 27, numéro 4, 1972

URI : https://id.erudit.org/iderudit/028349ar

DOI : https://doi.org/10.7202/028349ar

Aller au sommaire du numéro

Éditeur(s)

Département des relations industrielles de l'Université Laval

ISSN

0034-379X (imprimé)

1703-8138 (numérique)

Découvrir la revue

Citer ce compte rendu

Boivin, J. (1972). Compte rendu de [Collective Bargaining in Government (Readings and Cases) by J. Joseph Loewenberg and Michel H. Moskow, Englewood Cliffs, New Jersey, Prentice-Hall, Inc., 1972, 362 pp.] Relations industrielles / Industrial Relations, 27(4), 795-796.

https://doi.org/10.7202/028349ar

Tous droits réservés (C) Département des relations industrielles de l'Université Laval, 1972
Ce document est protégé par la loi sur le droit d'auteur. L'utilisation des services d'Érudit (y compris la reproduction) est assujettie à sa politique d'utilisation que vous pouvez consulter en ligne.

https://apropos.erudit.org/fr/usagers/politique-dutilisation/ 
publique parce que celle-ci est beaucoup plus diffuse que dans l'entreprise privée.

La dernière question traité par Lelchook et Lahne n'est pas la moindre. Il s'agit de la sécurité syndicale. En théorie, le principe de l'évaluation au mérite ne peut tolérer aucun mécanisme qui forcerait un fonctionnaire à faire partie d'un syndicat ou à payer une cotisation syndicale comme condition préalable à l'obtention ou au maintien d'un emploi dans la fonction publique. Pourtant, avec les progrès effectués par le syndicalisme, on retrouve dans certaines juridictions des clauses d'atelier syndical imparfait et, en de rares occasions aux Etats-Unis, des clauses de sécurité syndicale de type «formule Rand », même si dans la plupart des cas de telles clauses sont défendues par la loi. Pour les auteurs qui reconnaissent la contribution positive de ces clauses sur la stabilité de la relation employeur-syndicat, il semble que les clauses de type « formule Rand ( ( agency shop \) présentent un heureux compromis. Ils vont même jusqu'à prédire que de telles clauses connaîtront beaucoup de popularité dans un proche avenir. Si l'on se réfère à l'expérience du Québec qui constitue le laboratoire expérimental par excellence des relations du travail dans le secteur public en Amérique du Nord, ils ont sans doute raison.

\section{Jean BOIVIN}

Collective Bargaining in Government (Readings and Cases) by J. Joseph Loewenberg and Michel H. Moskow, Englewood Cliffs, New Jersey, Prentice-Hall, Inc., 1972, 362 pp.

Collective Bargaining in Government est un recueil de textes et de cas sur les relations du travail dans le secteur public des Etats-Unis. Ce livre ne présente aucun article nouveau mais il a le très grand avantage d'être un condensé des meilleurs ouvrages écrits sur le sujet au cours des cinq dernières années.

Le premier chapitre donne une idée d'ensemble du secteur public, tant du point de vue de l'évolution de l'emploi et de l'évolution de la syndicalisation que du cadre juridique dans lequel se dérou. lent les relations du travail. Il contient également un article de Neil W. Chamberlain qui fait très bien ressortir les différences entre le secteur public et le secteur privé.
Le second chapitre décrit les parties engagées dans le processus de négociation collective. Par exemple, on y retrouve des monographies de l'American Federation of Government Employees (AFGE), de l'American Federation of State, County and Municipal Employees (AFSCME), de l'American Federation of Teachers (AFT) et de la National Education Association (NEA), qui sont sans doute les quatre plus grandes organisations de travailleurs du secteur public aux Etats-Unis. Il y a également une description de certaines agences gouvernementales telle l'Office of Collective Bargaining (OCB) de la ville de New York ainsi qu'une discussion des arguments militant en faveur et contre la couverture des employés du secteur public par les mêmes organismes administratifs (v. g. Commission des relations du travail) qui ont juridiction sur le secteur privé.

Le chapitre troisième s'adresse au processus de négociation comme tel : détermination des unités de négociation, le champ de la négociation, le rôle des arbitres dans le règlement des griefs résultant de l'application des conventions collectives.

Le quatrième chapitre contient diverses études de cas se rapportant à des événements qui ont marqué l'histoire des relations du travail dans le secteur public américain: la grève des éboueurs de Memphis en 1968, au cours de laquelle le pasteur Martin Luther King fut assassiné ; la grève des policiers de Boston de 1919 ; la grève des employés des postes de 1970, etc.

Le cinquième chapitre présente un intérêt particulier pour les lecteurs familiers avec l'expérience québécoise car il traite des aspects politiques de la négociation dans le secteur public.

Quant au chapitre sixième, il fait le point sur le sujet très controversé aux Etats-Unis qu'est la grève, qu'elle soit légale ou illégale. Après avoir dressé un bilan de l'évolution du nombre de grèves entre 1958 et 1969, le chapitre six présente les meilleurs articles qui traitent des arguments favorables et défavorables au droit de grève pour les employés du secteur public. On y discute également de l'efficacité de certains substituts au droit de grève qui ont été essayés dans quelques états de même que des plus récentes propositions telles le \& Final Offer Selection Procedure $\gg$ dans lequel un arbitre 
n'aurait pas d'autre choix que de recommander la totalité des revendications de l'une ou l'autre des parties. Ce mécanisme fait actuellement l'objet d'étude par une commission d'enquête sénatoriale aux Etats-Unis et il est de plus en plus question que le Président Nixon propose qu'elle s'applique au secteur des transports et communications qui a été marqué par des grèves très coûteuses pour l'économie américaine au cours des dernières années.

Enfin, le dernier chapitre analyse différentes techniques de prévention et de solution des conflits telles la médiation, la conciliation, les commissions d'enquête factuelles (Fact Finding Boards), l'arbitrage obligatoire ou volontaire.

Un coup d'œil rapide à la liste des auteurs qui ont collaboré à ce volume permet d'établir hors de tout doute la qualité des ouvrages qu'il contient. Qu'il soit permis de mentionner, outre Neil W. Chamberlain, la collaboration de Arvid Anderson, Charles M. Rehmus, Arnold R. Weber, Sterling D. Spero, Milton Derber, Theodore W. Kheel, Jack Stieber, Jean T. McKelvey. Bref, il s'agit d'un ouvrage indispensable à toute personnes qui veut prétendre connaître quelque chose aux relations du travail dans le secteur public américain.

Jean BOIVIN

Manpower Planning and Programming, by Elmer $H$. Burack and James W. Walker, Boston, Allyn \& Bacon, Inc., 1972, $452 \mathrm{pp}$.

Cet ouvrage possède essentiellement deux caractéristiques: d'abord c'est un recueil d'études (plus de trente cinq). Ensuite il centre son attention sur la planification de main-d'œuvre au niveau micro-économique. «It describes in detail, within the context of technological, social and economic change, approaches for manpower planning and programming in large organizations ». En plus, il est à noter que cet ouvrage s'identifie comme un livre de base pour un cours universitaire, tant au niveau gradué que sousgradué, sur la planification de la maind'oeuvre au niveau de l'entreprise et aussi comme un complément pour un cours sur la direction du personnel.

Tout comme les relations industrielles, cette approche dite de main-d'oeuvre est multi-disciplinaire. A toute fin pratique on en est peut-être arrivé à substituer aux expressions de « relations industrielles » et de «direction du personnel» le qualificatif de main-d'œuvre. L'influence américaine sur ce point, l'impact de l'expression anglaise «manpower et la nouvelle vogue de «main-d'œuvre » peuvent expliquer qu'on étiquette maintenant une réalité assez vieille d'un nouveau nom.

Par contre, ce livre n'est pas un bouquin sur la direction du personnel mais plutôt sur cette partie de la direction du personnel qui se préoccupe de recrutement, de sélection, de promotion, de séparation et de planification. En un mot on se centre plus ici sur la sous-fonction de «staffing 》 de la fonction de direction de personnel, si on peut employer une expression de French.

Essentiellement les auteurs prétendent intégrer l'information éparse déjì existante sur cet aspect et présenter le concept de «manpower planning and programming dans un cadre logique nouveau.

Cet ouvrage comporte quatre parties principales: la première, intitulée «vue générale 》 décrit les récents changements technologiques, sociaux et économiques et leurs implications. En un mot cette partie constitue un arrière-plan qui permet au lecteur de mieux situer le problème. Dans un deuxième temps, on consacre trois chapitres pour expliquer le concept de planification de main-d'œuvre. Cette partie insiste sur des modèles de prévision de main-d'œuvre, sur des modèles de planification et sur des systèmes d'information sur la main-d'oeuvre.

Comme le proposait Vetter (qui malheureusement ne participe pas à cet ouvrage) dans une de ses publications, cette planification doit avoir un impact sur les différentes parties de la sous-fonction de «staffing 》. C'est effectivement ce que Burack et Walker font dans la troisième partie de cet ouvrage. Cependant, cette action qui doit suivre la planification est analysée ici en termes d'approches modernes à la programmation de la main-d'oeuvre. On examine ces approches pour le recrutement, le développement, les plans de carrières, les promotions et les remplacements, surtout le remplacement de cadres.

Enfin, les auteurs se concentrent sur quelques problèmes fondamentaux d'une telle planification de main-d'œuvre. 